\title{
c-Myc down-regulation is involved in proteasome inhibitor-mediated enhancement of radiotherapeutic efficacy in non-small cell lung cancer
}

\author{
JOOHEE JUNG ${ }^{1}$, EUN-JIN KIM ${ }^{2}$, HYE KYUNG CHUNG ${ }^{1}$, HEON JOO PARK ${ }^{3}$, \\ SEONG-YUN JEONG ${ }^{1}$ and EUN KYUNG $\mathrm{CHOI}^{1,2}$
}

${ }^{1}$ Institute for Innovative Cancer Research, ${ }^{2}$ Department of Radiation Oncology, Asan Medical Center, University of Ulsan
College of Medicine, Seoul; ${ }^{3}$ Department of Microbiology, College of Medicine, Inha University, Inchon, Republic of Korea

Received July 20, 2011; Accepted September 7, 2011

DOI: 10.3892/ijo.2011.1205

\begin{abstract}
In this study, the effect of MG132 (carbobenzoxylleucinyl-leucinyl-leucinal-H) at a low dose on radiotherapeutic efficacy and its accurate mechanism of radiosensitization were investigated in human non-small cell lung cancer. The effect of MG132 on ionizing radiation (IR)-induced cytotoxicity, cell proliferation and survival of A549 cells was evaluated. The protein expression modulated by MG132 and IR were inspected by Western blot analysis. To determine in vivo radiotherapeutic efficacy, tumor growth delay was analyzed in a A549 tumorbearing xenograft mouse model after single or repeated treatment of MG132 and/or IR. Induction of apoptosis and change of c-Myc expression in the tumor tissue was explored by histological analysis. MG132 at a non-toxic dose enhanced the radiationinduced cytotoxicity of A549 cells, accompanying a significant decrease of c-Myc expression. Suppression of c-Myc expression by small interfering RNA (siRNA) displayed enhancement of radiosensitivity similarly to MG132 treatment. Tumor growth in the xenograft mice was markedly delayed by systemic administration of MG132 combined with IR. In vivo down-regulation of c-Myc and increased induction of apoptosis were simultaneously observed in the tumor tissues followed by combinational treatment of MG132 and IR. The results reveal a novel mechanism for proteasome inhibitor-mediated radiosensitization in which c-Myc down-regulation is involved.
\end{abstract}

Correspondence to: Dr Eun Kyung Choi, Department of Radiation Oncology, Asan Medical Center, University of Ulsan College of Medicine, 86 Asanbyeongwon-gil, Songpa-gu, Seoul 138-736, Republic of Korea

E-mail: ekchoi@amc.seoul.kr

Dr Seong-Yun Jeong, Institute for Innovative Cancer Research, Asan Medical Center, University of Ulsan College of Medicine, 86 Asanbyeongwon-gil, Songpa-gu, Seoul 138-736, Republic of Korea E-mail: syj@amc.seoul.kr

Key words: c-Myc, proteasome inhibitor, fractionated radiation, radio-sensitizer, non-small cell lung cancer

\section{Introduction}

Non-small-cell lung cancer (NSCLC) is the leading cause of cancer-related deaths in sexual independent populations (1). Radiation therapy plays a significant role in the management of human lung cancer (2). However, radiation therapy is limited by the tolerance of the surrounding normal tissues. In addition, solid tumors tend to lessen the efficacy of radiation therapy owing to a hypoxic region (3). Due to these reasons, the overall cure rate and survival rate of patients remain low (4). To make up for these negative effects, the combination of anticancer drug with ionizing radiation (IR) is being used commonly for clinical applications. Particularly, for treatment of NSCLC, the chemoradiation therapy is reported to be beneficial and improve the survival rate (5). Furthermore, the combination therapy is able to reduce the usage dose and the toxicity. Many efforts for the screening and development of radiosensitizer have been reported to enhance the radiotherapeutic efficacy $(6,7)$. In order to improve a radiotherapeutic efficacy, the combination regimen of anticancer drug with IR has also been considered. The combination regimens representatively need to refine the administration route, dosage and sequencing (e.g., the chemotherapy given prior to IR or after IR, concurrent treatment and repeating treatment). Finally, the goal of the combination of chemotherapy and radiotherapy is the control of tumors and improvement of patient survival.

The proteasome plays a central role in the degradation of proteins related to the regulation of cell cycle, proliferation, apoptosis, angiogenesis, metastasis, and resistance to chemotherapy and radiotherapy (8). Proteasome inhibitors including bortezomib (boronic acid dipeptide derivative) and MG132 (carbobenzoxyl-leucinyl-leucinyl-leucinal-H) have anticancer activity $(9,10)$ and are known as a sensitizer to DNA-damaging agents, IR, and DNA cross linker for cancer therapy (11). The combination of bortezomib with IR induced nuclear factor- $\mathrm{\kappa B}$ (NF- $\kappa \mathrm{B})$ inhibition, apoptosis, and cytokines, which was associated with tumor control in head and neck cancer model (12), colorectal cancer model (13) as well as in androgen independent human prostate cancer cells (8). MG132 has been reported to sensitize PC-3 prostate cancer cells to IR by NF- $\kappa \mathrm{B}$ inhibition $(14,15)$ and improve the radiotherapeutic effect in NSCLC (16). 
In order to explain the mechanism of radiosensitizing effect, proteasome inhibitors including MG132 have been studied at high concentration $(\mu \mathrm{M})$ inducing apoptosis $(8,10,16)$, which might not be applicable to clinical treatment for human cancer.

In this study, we investigated the effect of MG132 on the radiotherapeutic efficacy in NSCLC A549 cells. We found a new mechanism of radiosensitization mediated by MG132 at a range of non-toxic dose (nM) in which down-regulation of c-Myc is involved. The effective therapeutic regimen for the combination of proteasome inhibitor and IR was also established in a mouse model bearing A549-derived tumors.

\section{Materials and methods}

Cell culture and treatment. A549 cells were maintained in Dulbecco's modified Eagle's medium (DMEM, Invitrogen, Grand Island, NY, USA) containing 10\% fetal bovine serum (FBS, Invitrogen) at $37^{\circ} \mathrm{C}$ in a humidified $5 \% \mathrm{CO}_{2}$ incubator. Cells were seeded in 96-well plates at a density of $2 \times 10^{3}$ cells/well one day prior to treatment. Cells were treated with MG132 (Calbiochem, San Diego, CA, USA) and irradiated $2 \mathrm{~h}$ later. Five pmole of c-Myc small interfering RNA (c-Myc siRNA, ON-TARGETplus SMARTpool L-003282, Dharmacon, Inc., Chicago, IL, USA) was transfected with Lipofectamine RNAiMax (Invitrogen, Carlsbad, CA, USA) $24 \mathrm{~h}$ prior to 5 Gy of IR.

Assays for cell viability. On the third day after treatment, cell cytotoxicity was assayed using Cell Counting Kit-8 (Dojindo, Kumamoto, Japan) according to the manufacturer's protocol. Data were expressed as the mean \pm standard deviation $(\mathrm{SD}, \mathrm{n}=8)$. For clonogenic assay, A549 cells were seeded into 6-well plate at concentrations of 50-1,000 cells/well depending on the dose of MG-132 and IR $24 \mathrm{~h}$ prior to MG132 treatment. After MG132 treatment, cells were irradiated from 0 to $10 \mathrm{~Gy}$. The cells were incubated for 12 days to allow colony formation, rinsed with phosphate buffered saline (PBS), fixed, and stained with $0.5 \%$ crystal violet solution in $10 \%$ methanol. The colonies composing $>50$ cells were counted, and the survival fraction for each treatment group was normalized to the surviving fraction from the untreated controls (plating efficiency). The dose enhancement ratio (DER) of IR (1) was calculated at the dose (Gy) for IR alone divided by the dose (Gy) for IR with drug, at a survival fraction of 0.1.

Western blot analysis. Cells at 80-90\% confluence were lysed in pro-prep solution (iNtRON Biotechnology, Seongnam, Korea). Proteins were separated by sodium dodecyl sulfate polyacrylamide gel electrophoresis (SDS-PAGE) through 10\% gel and transferred to polyvinylidene fluoride (PVDF) membrane. Membrane was blocked with 5\% skim milk (Becton-Dickinson, Co., Sparks, MD) in PBS containing 0.1\% Tween-20 (PBST) for $1 \mathrm{~h}$ at room temperature. Membrane was probed with either primary antibody against p53 (1:1000, Upstate, Temecula, CA), Cip1/WAF-1/p21 (1:1000, Upstate, Temecula, CA), c-Myc (1:1000, Abcam, Cambridge, UK) or GAPDH (1:5000, Assay designs, Ann Arbor, MI) overnight at $4^{\circ} \mathrm{C}$. Membrane was washed three times in PBST and incubated with peroxidase-conjugated mouse secondary antibody (1:2000, Jackson ImmunoResearch Laboratories, Inc., West Grove, PA) for 2 $\mathrm{h}$ at room temperature. Membrane was washed in PBST and the detection was done using enhanced chemiluminescence kit (ECL Western blotting detection reagents, GE Healthcare, Buckinghamshire, UK) and exposed to X-ray film (AGFA, Mortsel, Belgium).

Tumor growth delay. A549 cells ( $5 \times 10^{5}$ cells/mouse) were subcutaneously injected into hind limb of Balb/c nude mice (5-weeks old, SLC, Shizuoka, Japan). When tumors grew to $50 \mathrm{~mm}^{3}$, mice were randomized and divided into four groups ( $\mathrm{n}=5$ per group) such as untreated control, treated with MG132 alone, IR alone and MG132 combined with IR. MG132 (2.5 mg/kg) was intravenously injected $2 \mathrm{~h}$ prior to IR (5 Gy). Tumors were irradiated using $6 \mathrm{MV}$ photon beam linear accelerator (CL/1800, Varian, CA). In repeated treatment, MG132 (1.25 mg/kg) and IR (2 Gy) were administered once a week for 3 weeks. Tumor size was determined with a caliper every other day and tumor volume was calculated from the following formula; $\mathrm{V}=$ [longest dimension] $\mathrm{x}$ [shortest dimension $]^{2} \mathrm{x} 0.5$. To evaluate the anticancer efficacy of treatment, T/C (\%) on the final date of this experiment was calculated as follows: (change in tumor growth for treated group)/ (change in tumor growth for control group) x 100 .

Immunohistological analysis. Male Balb/c nude mice bearing A549 cell-derived tumors were intravenously injected with MG132 at $2.5 \mathrm{mg} / \mathrm{kg}$ and irradiated (2 Gy) once a week for 3 weeks. On the 21 st day, mice were sacrificed and the tumors were isolated, fixed in $4 \%$ paraformaldehyde and then embedded into paraffin. Sections of $5 \mu \mathrm{m}$ were prepared and rehydrated. To investigate c-Myc expression levels, tissue sections were incubated with c-Myc antibody (1:200, Abcam, Cambridge, UK) overnight at $4^{\circ} \mathrm{C}$. After washing, the tissue sections were incubated with biotin-conjugated anti-mouse antibody (1:100, Jackson ImmunoResearch Laboratories, Inc., West Grove, PA). After incubation with Vectastain elite ABC kit (Vector Laboratories, Inc., Burlingame, CA), the tissue sections were stained with DAB kit (Vector Laboratories, Inc.). The image on the tissue sections was observed and photographed with microscopy set (Olympus, Tokyo, Japan). To examine apoptosis, the tissue sections were incubated in distilled water for $1 \mathrm{~h}$ at $60^{\circ} \mathrm{C}$ and equilibrated with TdT labeling buffer for $10 \mathrm{~min}$. The tissue sections were incubated with TdT/biotinylated deoxyurine for $1 \mathrm{~h}$ at $37^{\circ} \mathrm{C}$. Donkey serum (10\%) was used to remove non-specific background. The apoptotic cells were visualized with ABC kit followed by DAB kit. The image was observed and photographed with a microscopy set.

\section{Results}

MG132 at a low dose sufficiently enhances radiosensitivity of A549 cells. To investigate whether MG132 at a dose so low as not to attenuate cell proliferation, could display a sufficient radiosensitization effect, we examined the cytotoxicity and clonogenicity in NSCLC A549 after treatment of IR and MG132 at various nano-molar concentrations. As shown in Fig. 1A, cell proliferation was not attenuated by $100 \mathrm{nM}$ or $400 \mathrm{nM}$ MG132. When combined with IR, $400 \mathrm{nM}$ MG132 significantly enhanced cytotoxicity both upon 5 and 10 Gy IR, although 100 nM MG132 showed no difference upon 5 Gy IR compared to control. The clonogenicity by the combination of $300 \mathrm{nM}$ MG132 with IR was markedly decreased (Fig. 1B). DER in A549 cells treated by combination of MG132 at 100 or $300 \mathrm{nM}$ with IR compared to 
A

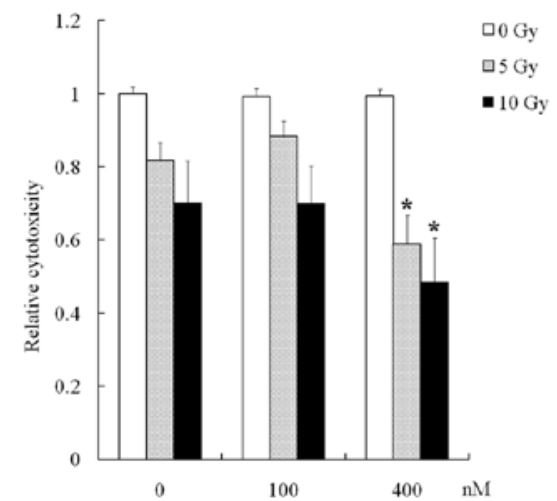

B

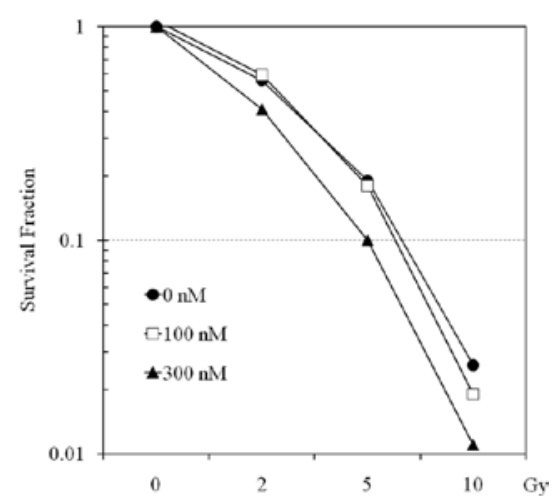

Figure 1. Proteasome inhibitor MG132 enhances radiosensitivity. (A) Cytotoxicity assay. A549 cells were treated with MG132 for $2 \mathrm{~h}$ prior to IR. After incubation for $72 \mathrm{~h}$, cell viability was measured as described in Materials and methods. The viability of untreated cells was normalized to ' 1 ' and relative cytotoxicity was plotted. Data presented the mean \pm SD (" $p<0.01 \mathrm{t}$-test). (B) Clonogenic assay. A549 cells were treated with MG132 for $2 \mathrm{~h}$ prior to IR. The cells were incubated for 12 days to allow colony formation. The colonies composing $>50$ cells were counted, and plotted.

A

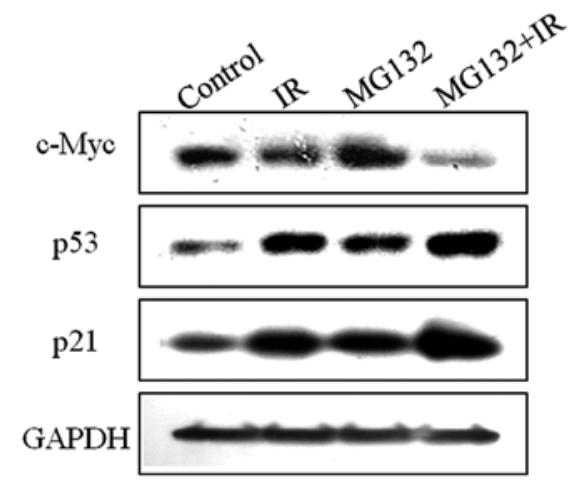

B

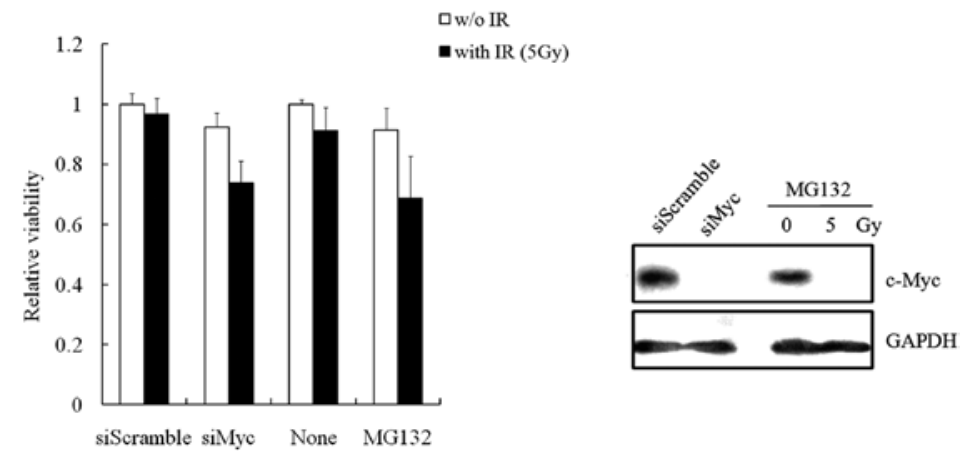

Figure 2. c-Myc down-regulation is involved in MG132-mediated radiosensitization. (A) A549 cells treated with MG132 (300 nM) and/or IR (5 Gy) were harvested after $48 \mathrm{~h}$. Protein expression was investigated by Western blot analysis. (B) A549 cells were transfected with c-Myc siRNA (siMyc, 5 pmole) or scrambled siRNA as a negative control (siScramble, 5 pmole) and incubated for $24 \mathrm{~h}$. Otherwise, cells were treated with MG132 (400 nM) for $2 \mathrm{~h}$. At $72 \mathrm{~h}$ after 5 Gy of IR, cell viability was measured (left panel). The viability of untreated cells was normalized to ' 1 ' and relative cytotoxicity was plotted. Data presented the mean $\pm \mathrm{SD}\left({ }^{*} \mathrm{p}<0.05 ;{ }^{* * *} \mathrm{p}<0.001 \mathrm{t}\right.$-test). The level of c-Myc expression was determined by Western blot analysis (right panel).

IR alone was 1.06 and 1.36 , respectively at a survival fraction of 0.1. These results demonstrated that MG132 at a range of non-toxic dose (nM) sufficiently enhanced the radiosensitivity of A549 cells.

c-Myc down-regulation is involved in MG132-mediated radiosensitization. To study the molecular mechanism of radiosensitization effect mediated by MG132, we performed Western blot analysis against key molecules involved in cell death and proliferation in A549 cells treated with MG132 and/ or IR (Fig. 2A). The expression of p53 and p21 was increased by either IR or MG132, corresponding to the activation of ataxia telangiectasia mutated (ATM)-p53 signaling pathway by IR or 
A

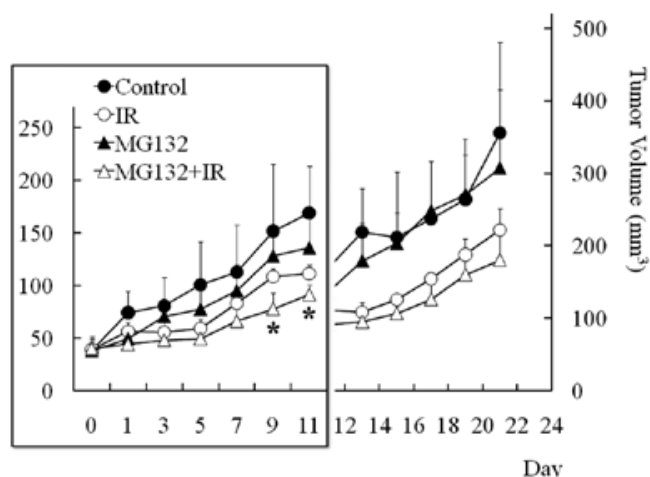

B

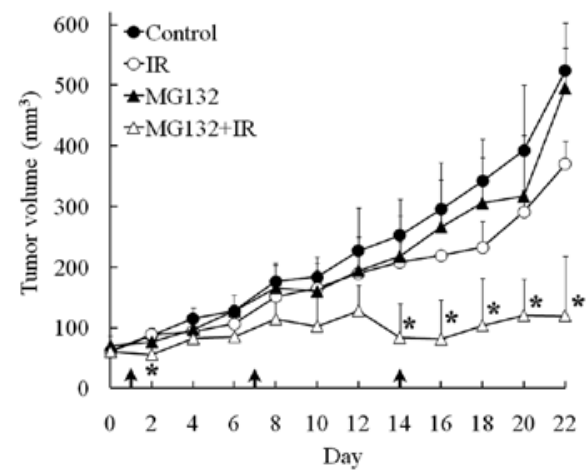

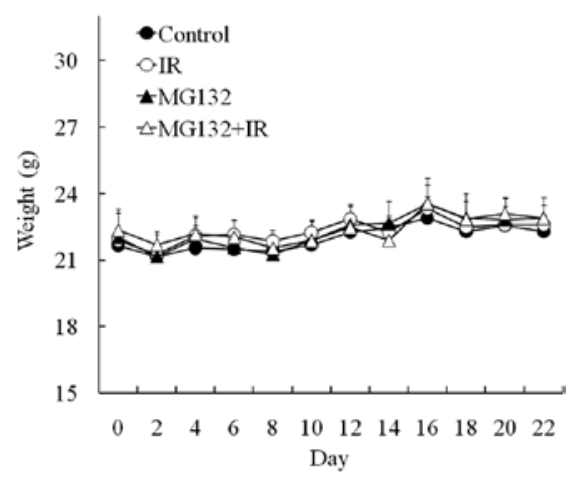

Figure 3. MG132 enhanced the radiotherapeutic efficacy. (A) Single treatment. MG132 (2.5 mg/kg) was intravenously injected $2 \mathrm{~h}$ prior to IR at $5 \mathrm{~Gy}$ into mice bearing A549-derived tumor, and then irradiated. (B and C) Repeated treatment. Mice bearing A549-derived tumor were treated with MG132 (1.25 mg/kg) and IR at 2 Gy once a week for 3 weeks. Tumor sizes and body weights were determined every other day. Data are presented as the mean \pm SD ( $\mathrm{p}<0.05 \mathrm{t}$-test).

the inhibition of protein degradation by MG132. Intriguingly, c-Myc expression was notably decreased in the cells treated with both MG132 and IR, while it was not decreased by MG132 or IR alone. To assure that the down-regulation of c-Myc plays a role in the radiosensitization, cells were transfected with c-Myc siRNA instead of MG132 treatment and cell viabilities were compared to that of MG132-treated cells. As shown in Fig. 2B, cell viability decreased to $73 \%$ by the combination of c-Myc siRNA with IR, which was similar to the decrease of cell viability by the combination of MG132 with IR. The sufficient suppression of c-Myc expression was confirmed by Western blot analysis. These results suggested that c-Myc down-regulation played a key role to cause MG132-mediated radiosensitization.

Repeated administration of low-dose MG132 enhanced the radiotherapeutic efficacy in vivo. To evaluate the in vivo radiosensitization effect of MG132, mice bearing A549-derived tumor were intravenously injected with MG 132 and the tumor was exposed to IR. In mice treated once with $2.5 \mathrm{mg} / \mathrm{kg}$ of MG132 and 5 Gy of IR, the tumor growth was more delayed than that of mice treated with 5 Gy alone (Fig. 3A and Table I). During the first 2 weeks, the difference in growth rate was especially apparent between these two groups. On the other study, mice were repeatedly treated with MG132 and/or IR. MG132 at $1.25 \mathrm{mg} / \mathrm{kg}$ was intravenously injected $2 \mathrm{~h}$ prior to IR (2 Gy),
Table I. Summary of in vivo efficacy.

\begin{tabular}{llcllc}
\hline & \multicolumn{2}{c}{ Single treatment } & & \multicolumn{2}{c}{ Repeating treatment } \\
\cline { 2 - 3 } \cline { 5 - 6 } Group & $\%$ T/C & $\begin{array}{c}\text { Tumor growth } \\
\text { delay (\%) }\end{array}$ & & T/C & $\begin{array}{c}\text { Tumor growth } \\
\text { delay (\%) }\end{array}$ \\
\hline Control & 100 & 100 & & 100 & 100 \\
IR & 57.6 & 202 & & 75.3 & 129 \\
MG132 & 84.9 & 135 & & 97.3 & 112 \\
MG132+IR & $44.2^{\mathrm{a}}$ & 254 & & $30.5^{\mathrm{a}}$ & 261 \\
\hline
\end{tabular}

$\mathrm{T} / \mathrm{C}$, tumor growth inhibition. ${ }^{\mathrm{a}} \mathrm{p}<0.01 \mathrm{vs}$. IR.

and the treatment was repeated weekly for 3 weeks (Fig. 3B). The group treated with either MG132 or IR alone showed moderately delayed tumor growth from that of untreated group. Notably, the group treated with both MG132 and IR manifested significantly delayed tumor growth during the experimental period. At the final day, the tumor volume of control group and the group treated with MG132 and IR reached 524 \pm 78 and $119 \pm 97 \mathrm{~mm}^{3}$, respectively. No difference in weights among the groups was not observed during the entire experimental period, 
A

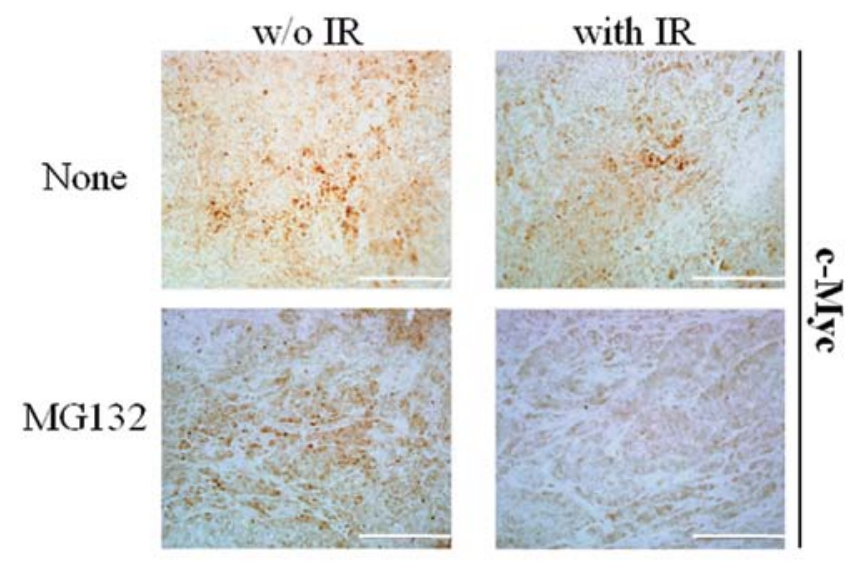

B

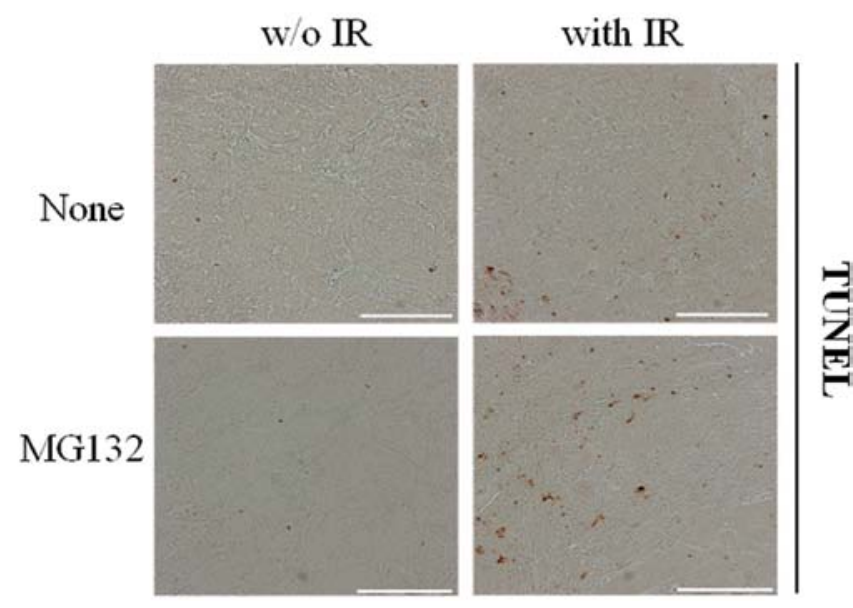

Figure 4. Down-regulation of c-Myc and apoptosis were correlatively induced by the combination of MG132 and IR. (A) Immunohistochemistry for c-Myc expression. Mice bearing A549-derived tumor were treated with MG132 $(2.5 \mathrm{mg} / \mathrm{kg}$, i.v.) and $2 \mathrm{~h}$ later the tumor was irradiated $(2 \mathrm{~Gy})$. The treatment was repeated once a week for 3 weeks. On the 21st day, the tumors were isolated and immunostaining was performed against c-Myc as described in Materials and methods. (B) TUNEL assay. Tissues were prepared as in (A). Apoptotic cells in the tissue were visibilized by TUNEL staining. Scale bar, $50 \mu \mathrm{m}$.

indicating that toxicity was negligible even though MG132 was repeatedly administered (Fig. 3C).

As shown in Table I, the quantitative evaluation of in vivo anticancer efficacy displayed that the repeated treatment with MG132 and IR had greater efficacy for tumor growth inhibition (\% T/C, 30.5) than the single treatment (\% T/C, 44.2). Tumor growth was delayed by the repeated treatment to $261 \%$, which was also more effective than that of the single treatment $(254 \%)$. MG132 alone did not show significant anticancer efficacy regardless of the single or repeated treatment. The anticancer efficacy of single shot of IR at 5 Gy was better than that of three times fractionated IR at 2 Gy. Nonetheless that either the repeated treatment of MG132 alone or fractionated shot of IR at 2 Gy showed little valuable anticancer efficacy, combination of these two regimens displayed the greatest anticancer efficacy. Thus, these results indicated that the low dose MG132 effectively sensitizes A549-derived xenograft tumors to IR, especially when it was treated repeatedly.

Down-regulation of c-Myc and apoptosis in tumor tissue were correlatively induced by the combination of MG132 and IR. For further investigation of MG132-mediated in vivo radiosensitization, tumor tissues from the mice treated with MG132 and/or IR were immunohistochemically analyzed. As shown in Fig. 4A, c-Myc protein was abundantly expressed in A549derived xenograft tumor tissue. The expression level of c-Myc was not affected by either MG132 or IR alone, while it was obviously down-regulated by the combination of MG132 and IR. Furthermore, IR-induced apoptosis in the tumor tissue was augmented by the combination with MG132 (Fig. 4B), although MG132 alone did not induce apoptosis. These histological results reflected that the combination of MG132 with IR induced the c-Myc down-regulation and apoptosis correlatively, thereby radiotherapeutic efficacy was potentiated.

\section{Discussion}

In this study, we investigated the effectiveness and mechanism of MG132 for radiosensitization both in vitro and in vivo. We determined MG132 at a range of low dose (nM) displayed sufficient radiosensitization effect both in vitro and in vivo. In human lung cancer A549 cells, the concentration of MG132 which did not show much cellular damage by itself significantly enhanced the cytotoxicity and decreased the clonogenicity mediated by exposure to IR. The induction of apoptosis in A549-derived xenograft tumor tissue treated with MG132 and IR together was apparently increased, and its tumor growth was delayed. We found that the effect of radiosensitization mediated by MG132 was exerted through the mechanism in which down-regulation of c-Myc is involved. c-Myc downregulation was observed upon the study of gene expression patterns in the lung cancer cells, which is related to cell death and cell arrest. The expression level of c-Myc in A549-derived xenograft tumor tissue treated with MG132 and IR was obviously down-regulated and the apoptosis was correlatively augmented. The c-Myc protein which was down-regulated by treatment of MG132 and IR is known as a potent regulator of cell growth, proliferation, differentiation and apoptosis $(17,18)$. Although the mechanism of c-Myc down-regulation mediated by the combination of MG132 with IR has not been accurately defined in this study, a possible involvement of $\beta$-catenin could be suggested according to a recent report (19) describing that the cleavage of $\beta$-catenin by MG132 is associated with decrease in the mRNA expression of c-myc.

Following exposure of cells to IR, it has been reported that DNA double strand break (DSB) and G2/M cell cycle arrest occur (20), leading to mitotic cell death and apoptosis (21). In irradiated cells, the activation of ATM kinase that phosphorylates p53, checkpoint kinase 1 (Chk1), Chk2 and H2AX plays an important role in IR-induced DNA damage response (22-25). Increase of p53 as well as p21, a p53-regulated gene, was observed in cells treated with IR and they were more accumulated by additional treatment of MG132. Suppression of c-Myc by siRNA in A549 cells, mimics the down-regulation of c-Myc by treatment of MG132, showing enhanced radiotherapeutic effect both in vitro and in vivo. These results suggest that c-Myc 
down-regulation is related to improved radiotherapeutic efficacy. For a mechanism of radiosensitization by MG132, c-Myc down-regulation might coordinately facilitate the increase of p21 through induction of transforming growth factor-beta (TGF- $\beta$ ). Activation of p53 in ATM signaling pathways by IR is importantly related with TGF- $\beta$ that regulates cell-growth, differentiation, apoptosis and angiogenesis for suppression of malignancy and tumor progression. The induction of TGF- $\beta$ by IR inhibited c-Myc and increases p21, consequently resulting in the induction of apoptosis (26). Additionally, it has been reported that c-Myc directly induces the gene encoding the transcription factor $\mathrm{AP} 4$, which binds to recognition motifs located in p21 promoter and medicates transcriptional repression of p21 (27). The accumulated p21 in human lung cancer cells by the combination of MG132 with IR treatment shown in our experiment might be due to both c-Myc-induced AP4 elevation and the activation of the ATM-p53 pathway by IR, thus c-Myc down-regulation and prolonged p21 activity might exert the radiosensitization effect.

In this study, an effective therapeutic regimen for the combination of proteasome inhibitor and IR was also established in mouse model bearing A549 (NSCLC)-derived xenograft tumor. The new regimen using the fractionated radiation with low-dose MG132 displayed notable enhancement of radiotherapeutic efficacy. While the effect of tumor growth delay of single treatment was maintained only for 2 weeks, the fractionated radiation with MG132 markedly delayed the tumor growth over 3 weeks without significant observation of toxicity. The results might be due to that the activity of MG132, which is quickly reversible, although it has a potent activity as proteasome inhibitor in a broad spectrum. The short preservation of MG132 activity for radiosensitization was effectively compromised by repeated treatment with low dosage of MG132 and IR without adverse effects. This result suggests the feasibility of using reduced dose as low as clinically applicable, in contrast with the usage of high dose of MG132 and IR previously suggested (16). Our results from this study might be able to provide helpful information to improve radiotherapeutic efficacy for the treatment of human lung cancer.

\section{Acknowledgements}

This work was supported by a grant of the Korea Health 21 R\&D Project, Ministry for Health and Welfare, Republic of Korea (A062254), a grant of the Korean Health Technology R\&D Project, Ministry for Health and Welfare, Republic of Korea (A102059), the Nuclear R\&D program through the Korea Science and Engineering Foundation funded by the Ministry of Education, Science and Technology of Korea (2008-03876) and the Basic Science Research Program through the National Research Foundation of Korea (NRF) funded by the Ministry of Education, Science and Technology (313-2008-2-E00444).

\section{References}

1. Jemal A, Thun MJ, Ries LA, et al: Annual report to the nation on the status of cancer, 1975-2005, featuring trends in lung cancer, tobacco use, and tobacco control. J Natl Cancer Inst 100: 1672$1694,2008$.

2. O'Rourke N, Roque IFM, Farre Bernado N and Macbeth F: Concurrent chemoradiotherapy in non-small cell lung cancer. Cochrane Database Syst Rev 6: CD002140, 2010.
3. Overgaard J: Hypoxic radiosensitization: adored and ignored. J Clin Oncol 25: 4066-4074, 2007.

4. Dillman RO, Seagren SL, Propert KJ, et al: A randomized trial of induction chemotherapy plus high-dose radiation versus radiation alone in stage III non-small-cell lung cancer. N Engl J Med 323: 940-945, 1990.

5. Sandler A: State-of-the-art treatment for advanced non-smallcell lung cancer. Oncology 17: 15-22, 2003.

6. Choi EK, Terai K, Ji IM, et al: Upregulation of NAD(P)H:quinone oxidoreductase by radiation potentiates the effect of bioreductive beta-lapachone on cancer cells. Neoplasia 9: 634-642, 2007.

7. Jeong SY,Park SJ, Yoon SM, et al: Systemic delivery and preclinical evaluation of Au nanoparticle containing beta-lapachone for radiosensitization. J Control Release 139: 239-245, 2009.

8. Goktas S, Baran Y, Ural AU, et al: Proteasome inhibitor bortezomib increases radiation sensitivity in androgen independent human prostate cancer cells. Urology 75: 793-798, 2010.

9. Teicher BA, Ara G, Herbst R, Palombella VJ and Adams J: The proteasome inhibitor PS-341 in cancer therapy. Clin Cancer Res 5: 2638-2645, 1999.

10. Han YH, Moon HJ, You BR and Park WH: The attenuation of MG132, a proteasome inhibitor, induced A549 lung cancer cell death by p38 inhibitor in ROS-independent manner. Oncol Res 18: 315-322, 2010.

11. Orlowski RZ and Kuhn DJ: Proteasome inhibitors in cancer therapy: lessons from the first decade. Clin Cancer Res 14: 1649-1657, 2008.

12. Van Waes C, Chang AA, Lebowitz PF, et al: Inhibition of nuclear factor-kappaB and target genes during combined therapy with proteasome inhibitor bortezomib and reirradiation in patients with recurrent head-and-neck squamous cell carcinoma. Int $\mathbf{J}$ Radiat Oncol Biol Phys 63: 1400-1412, 2005.

13. Russo SM, Tepper JE, Baldwin AS Jr, et al: Enhancement of radiosensitivity by proteasome inhibition: implications for a role of NF-kappaB. Int J Radiat Oncol Biol Phys 50: 183-193, 2001.

14. Pajonk F, van Ophoven A, Weissenberger C and McBride WH: The proteasome inhibitor MG-132 sensitizes PC-3 prostate cancer cells to ionizing radiation by a DNA-PK-independent mechanism. BMC Cancer 5: 76, 2005.

15. Warren G, Grimes K, Xu Y, Kudrimoti M and St Clair W: Selectively enhanced radiation sensitivity in prostate cancer cells associated with proteasome inhibition. Oncol Rep 15: 1287-1291, 2006.

16. Grimes KR, Daosukho C, Zhao Y, Meigooni A and St Clair W: Proteasome inhibition improves fractionated radiation treatment against non-small cell lung cancer: an antioxidant connection. Int J Oncol 27: 1047-1052, 2005.

17. Meyer N and Penn LZ: Reflecting on 25 years with MYC. Nat Rev Cancer 8: 976-990, 2008.

18. Pelengaris S, Khan M and Evan G: c-MYC: more than just a matter of life and death. Nat Rev Cancer 2: 764-776, 2002.

19. Cervello M, Giannitrapani L, La Rosa M, et al: Induction of apoptosis by the proteasome inhibitor MG132 in human HCC cells: Possible correlation with specific caspase-dependent cleavage of beta-catenin and inhibition of beta-catenin-mediated transactivation. Int J Mol Med 13: 741-748, 2004.

20. Iliakis G, Wang Y, Guan J and Wang H: DNA damage checkpoint control in cells exposed to ionizing radiation. Oncogene 22: 5834-5847, 2003.

21. Dewey WC, Ling CC and Meyn RE: Radiation-induced apoptosis: relevance to radiotherapy. Int J Radiat Oncol Biol Phys 33: 781-796, 1995.

22. Ahn JY, Schwarz JK, Piwnica-Worms H and Canman CE: Threonine 68 phosphorylation by ataxia telangiectasia mutated is required for efficient activation of Chk 2 in response to ionizing radiation. Cancer Res 60: 5934-5936, 2000.

23. Fernandez-Capetillo O, Chen HT, Celeste A, et al: DNA damageinduced G2-M checkpoint activation by histone H2AX and 53BP1. Nat Cell Biol 4: 993-997, 2002.

24. Lavin MF, Birrell G, Chen P, Kozlov S, Scott S and Gueven N: ATM signaling and genomic stability in response to DNA damage. Mutat Res 569: 123-132, 2005.

25. Matsuoka S, Huang M and Elledge SJ: Linkage of ATM to cell cycle regulation by the Chk2 protein kinase. Science 282 : 1893-1897, 1998.

26. Dancea HC, Shareef MM and Ahmed MM: Role of radiationinduced TGF-beta signaling in cancer therapy. Mol Cell Pharmacol 1: 44-56, 2009.

27. Jung $\mathrm{P}$ and Hermeking $\mathrm{H}$ : The c-MYC-AP4-p21 cascade. Cell Cycle 8: 982-989, 2009. 